\title{
Growth and Integrability in the Dynamics of Mappings
}

\author{
Alexander P. Veselov \\ Department of Mathematics and Mechanics, Moscow State University, SU-119899 Moscow, USSR
}

Received August 12, 1991

\begin{abstract}
The growth of some numerical characteristics of the mappings under their iterations in the context of the general problem of integrability is discussed. In the general case such characteristics as complexity by Arnold or the number of the different images for the multiple-valued mappings are growing exponentially. It is shown that the integrability is closely related with the polynomial growth. The analogies with quantum integrable systems are discussed.
\end{abstract}

The goal of this paper is to discuss the growth of some concrete numerical characteristics of the mapping under its iterations in the context of the general problem of integrability for such discrete systems (see e.g. [1]). The results can be summarized in a quite natural way: The integrability has an essential correlation with the weak growth of certain characteristics. One of them is the complexity introduced and investigated by Arnold in the recent papers [2]. In the simplest case for the mappings $f$ of the plane the complexity can be defined as the number of intersection points of the fixed curve $\Gamma_{1}$ with the image of the second curve $\Gamma_{2}$ under the $k^{\text {th }}$ iteration of $f$

$$
A_{\Gamma_{1}, \Gamma_{2}}^{f}(k)=\# \Gamma_{1} \cap f^{(k)}\left(\Gamma_{2}\right) .
$$

If the mapping $f$ is the polynomial one and the curves $\Gamma_{1}$ and $\Gamma_{2}$ are algebraic, then it is easy to see that the growth of $A_{\Gamma_{1}, \Gamma_{2}}^{f}(k)$ will in general exponential on $k$, what is in a good agreement with the general Arnold's result [2]. In the first paragraph we will show that for the integrable (in the various senses) polynomial automorphisms of the plane this growth is much weaker

$$
A_{\Gamma_{1}, \Gamma_{2}}^{f}(k)<C\left(\Gamma_{1}, \Gamma_{2}, f\right),
$$

and this property turns out to be characteristic for the integrable mappings in this case. In the rest of the paper we discuss the dynamics of the multiple-valued mappings (correspondences) $\Phi$ and the growth of the numbers $N_{x}^{\Phi}(k)$ of the different images 
under the $k^{\text {th }}$ iteration of $\Phi$

$$
N_{x}^{\Phi}(k)=\# \Phi^{(k)}(x) .
$$

The second paragraph is devoted to the symplectic correspondences $\Phi$, which are integrable in the Liouville sense, i.e. have sufficiently many involutive integrals. It is shown that the numbers $N_{x}^{\Phi}(k)$ for them grow polynomially on $k$ instead of usual exponential growth (see also [3]). In the last paragraph we try to understand what the polynomial growth of $N_{x}^{\Phi}(k)$ means for the dynamics of algebraic correspondences $\Phi: \mathbb{C} P^{1} \rightarrow \mathbb{C} P^{1}$. The partial results we present also demonstrate the connection of this property with the integrability. This case is very interesting because of various connections and parallels with the theory of quantum integrable systems and quantum groups. Notice that the role of the well-known Yang-Baxter equation in the approach to the theory of quantum groups developed by Faddeev and his collaborators is to supply a certain growth of some related algebra (analogue of Poincaré-Birkhoff-Witt theorem). We hope that the results about the dynamics of the algebraic correspondences can be used for the construction of the new solutions of Yang-Baxter equation and integrable quantum models.

This paper was completed when the author was the guest of the Forschungsinstitut für Mathematik (ETH, Zürich). I am grateful to Prof. J. Moser and E. Zehnder for this possibility and stimulating discussions.

\section{Integrable Polynomial Automorphisms of the Plane and the Complexity by Arnold}

Let $f(x, y)=(P(x, y), Q(x, y))$ be some polynomial mapping $\mathbb{C}^{2} \rightarrow \mathbb{C}^{2}$, the inverse for which also is polynomial one. Such mappings form affine Cremona group $G A_{2}(\mathbb{C})$.

The problem about iterations of such a mapping $f$ can be considered as the solution of the following difference system:

$$
\left\{\begin{array}{l}
x_{k+1}=P\left(x_{k}, y_{k}\right) \\
y_{k+1}=Q\left(x_{k}, y_{k}\right) .
\end{array}\right.
$$

We will suppose that $f$ as an element of $G A_{2}$ has an infinite order otherwise the problem about its dynamics is not arising.

Following [4], let us give the

Definition. The mapping $g \in G A_{2}$ is called a symmetry of the dynamical system (1.1) if $g$ commutes with $f: f \circ g=g \circ f$. We say that symmetry $g$ is nontrivial, if $g$ generates with $f$ the subgroup $\mathbb{Z} \oplus \mathbb{Z} \subset G A_{2}$.

If $\left(x_{k}, y_{k}\right)$ is a solution of the system (1.1), then $\left(x_{k}^{\prime}, y_{k}^{\prime}\right)=g\left(x_{k}, y_{k}\right)$ is also the solution of (1.1), because of the commutativity $f$ and $g$. It means that $g$ transforms the set of the solutions of (1.1) into itself. So this definition is in a good agreement with the traditional symmetry approach arising to Lie (modern development and application of this aproach for the classification of integrable nonlinear partial differential equations, see in [5]). To the author's opinion this approach to the integrability is the most apropriate for the dynamics of mappings (see [1]).

Now let us consider two algebraic curves $\Gamma_{1}, \Gamma_{2}$ in the plane and the intersection of $\Gamma_{2}$ with the image of $\Gamma_{1}$ under the $k^{\text {th }}$ iteration of $f$

$$
A_{\Gamma_{1}, \Gamma_{2}}^{f}(k)=\# \Gamma_{2} \cap f^{(k)}\left(\Gamma_{1}\right) .
$$


It is the particular case of the numerical characteristics introduced and investigated by Arnold [2], which he calls complexity. It is closely connected with such characteristics as the algebraic degrees of the iterations of $f$, where an algebraic degree of the polynomial mapping $f=(P, Q)$ is defined as the maximum of the full degrees of its components

$$
\operatorname{deg} f=\max (\operatorname{deg} P, \operatorname{deg} Q) .
$$

Theorem 1. The following 4 conditions are equivalent to each other:

1) the dynamical system (1.1) has a nontrivial symmetry;

2) there exists a polynomial change of coordinates transforming the mapping $f$ to the triangular form:

$$
f(x, y)=(a x+P(y), b y+c),
$$

where $P(y)$ is some polynomial of $y$;

3) the algebraic degrees of all iterations of the mapping $f$ are bounded:

$$
\operatorname{deg} f^{(k)}<C(f)
$$

4) the complexity $A_{\Gamma_{1}, \Gamma_{2}}^{f}(k)$ is bounded:

$$
A_{\Gamma_{1}, \Gamma_{2}}^{f}(k)<C\left(\Gamma_{1}, \Gamma_{2}, f\right)
$$

for all $\Gamma_{1}, \Gamma_{2}$ being in the general position (such that these intersections consist of the finite numbers of points).

Proof. The equivalence 1) $\Leftrightarrow 2$ ) $\Leftrightarrow 3$ ) was established in [4] and simply follows from the algebraic results about affine Cremona group $G A_{2}[6,7]$. The theorem by Jung [6] plays a fundamental role here; it states that this group is the amalgamated free product of the groups of affine and the triangular transformations

$$
G A_{2}=A \underset{A \cap T}{*} T .
$$

Using this theorem Wright gave some description of all abelian subgroups in $G A_{2}$ [7]. As follows from his results all subgroups $\mathbb{Z} \oplus \mathbb{Z}$ are conjugated in $G A_{2}$ to some subgroups of $A$ or $T$, and therefore the condition (1.5) holds. Moreover he proved that this condition is sufficient to the conjugacy of the element $f \in G A_{2}$ to some element of $A$ or $T$. To establish the property 2 ) notice that over $\mathbb{C}$ any affine transformation can be transformed to the triangular form. It is easy to check that any triangular transformation of infinite order has nontrivial symmetry. It leads to the equivalence of the first three conditions.

Now the boundness of the complexity follows from the inequality

$$
A_{\Gamma_{1}, \Gamma_{2}}^{f}(k) \leq \operatorname{deg} P_{1} \cdot \operatorname{deg} P_{2}\left(\operatorname{deg} f^{(k)}\right),
$$

where $P_{1}=0, P_{2}=0$ are the equations of the curves $\Gamma_{1}$ and $\Gamma_{2}$. Indeed the intersection $\Gamma_{1} \cap f^{(k)}\left(\Gamma_{2}\right)$ is described by the system

$$
\left\{\begin{aligned}
P_{1}(x, y) & =0 \\
P_{2}\left(f^{(k)}(x, y)\right) & =0,
\end{aligned}\right.
$$

which according to the well-known Bezout theorem has no more than

$$
\operatorname{deg} P_{1}(x, y) \operatorname{deg} P_{2}\left(f^{(k)}(x, y)\right) \leq \operatorname{deg} P_{1} \cdot \operatorname{deg} P_{2} \cdot \operatorname{deg} f^{(k)}
$$


solutions, otherwise we will have the whole common curve and not a general position. The inverse implication 4) $\Rightarrow 3$ ) follows from the fact that for any mapping $h \in G A_{2}$ there exists an open by Zariski set of the pairs of the straight lines $\left(l_{1}, l_{2}\right)$, such that

$$
\# l_{2} \cap h\left(l_{1}\right)=\operatorname{deg} h .
$$

The proof is finished.

Remark 1. If we add in the condition 2) to the triangular transformations the affine ones then we will have the implications 1) $\Leftrightarrow 2) \Leftrightarrow 3$ ) $\Rightarrow 4$ ) for the real affine Cremona group $G A_{2}(\mathbb{R})$ (see $[4,7]$ ). We think that the implication 4) $\Rightarrow 3$ ) also holds on the reals but have not now the complete proof.

Remark 2. The mapping $f \in G A_{2}$ of the finite order: $f^{(N)} \equiv$ Id for some $N$, is conjugated to the triangular or affine form, as follows from [7].

Notice that the iterations of affine and triangular transformations can be found explicitly. Thus, the conditions 1), 2), meaning the integrability of the dynamical system (1.1), imply the boundness of the complexity by Arnold for this system instead of usual exponential growth.

It is very interesting to compare these considerations with ones by Moser [8], who analyzed the mapping

$$
f:\left(\begin{array}{l}
x \\
y
\end{array}\right) \rightarrow\left(\begin{array}{l}
\left(x+y^{3}\right) \cos \alpha-y \sin \alpha \\
\left(x+y^{3}\right) \sin \alpha+y \cos \alpha
\end{array}\right), \quad \sin \alpha \neq 0
$$

It is easy to see that the algebraic degrees of $f$ grow exponentially

$$
\operatorname{deg} f^{(k)}=3^{k}
$$

and therefore this $f$ is nonintegrable in our sense. Moser has proved the nonintegrability of this mapping in the Birkhoff sense, i.e. the divergence of the series transforming this mapping to the normal form. His investigation is based on the calculation of the numbers of the periodic points and also uses the algebraic nature of the maping.

Similar properties have the quadratic mappings from $G A_{2}$. By affine change of coordinates such mappings can be reduced to the form

$$
f\left(\begin{array}{l}
x \\
y
\end{array}\right)=\left(\begin{array}{c}
\alpha_{0}+\alpha_{1} x+\alpha_{2} y+\alpha x^{2} \\
\beta_{0}+\beta_{1} x+\beta_{2} y+\beta x^{2}
\end{array}\right),
$$

where $\alpha_{1} \beta_{2}-\alpha_{2} \beta_{1} \neq 0, \alpha \beta_{2}-\alpha_{2} \beta=0$. It is easy to see that

$$
\operatorname{deg} f^{(k)}=2^{k}, \quad \text { if } \quad \alpha \neq 0,
$$

so the integrability has a place only for $\alpha=0$. This leads to two cases:

1) $\alpha_{2}=0$; 2) $\beta=0$. The first case is triangular, in the second we have affine transformation.

In particular, Henon mapping

$$
\left(\begin{array}{l}
x \\
y
\end{array}\right) \rightarrow\left(\begin{array}{c}
1+y-a x^{2} \\
b x
\end{array}\right)
$$

is integrable in our sense only for $a=0$.

In the symplectic case we can pose another question: whether or not a given polynomial automorphism from $G A_{2}$ has a polynomial integral? 
Using Theorem 1 and some results of the paper [10] by Friedland and Milnor one can prove (see $[4,11]$ ) that the existence of a nonconstant polynomial integral $I$ for the dynamical system (1.1): $I\left(x_{k+1}, y_{k+1}\right) \equiv I\left(x_{k}, y_{k}\right)$ for the symplectic $f$ is equivalent to every condition from 1 ) -4 ) of Theorem $1^{1}$. To replace the polynomial integral by a rational one changes nothing in this statement.

So the situation with polynomial automorphisms of $\mathbb{C}^{2}\left(\mathbb{R}^{2}\right)$ seems to be clear. To investigate analogous problems for other dimensions $n>2$ is an interesting open problem. We mention here only the recent paper by Moser [12], where the quadratic symplectic mappings are considered.

\section{Multiple-valued Symplectic Mappings: The Growth of the Numbers of Different Images of Their Iterations}

We begin with the remark that for the symplectic mappings the multiple-valueness is not exotic but is quite a natural property. Let us consider, for example, the symplectic mapping

$$
\Phi: T^{*} M^{n} \rightarrow T^{*} M^{n}, \quad \Phi(p, q)=(P, Q),
$$

determined by the relations

$$
P=\frac{\partial \mathscr{L}}{\partial Q}, \quad p=-\frac{\partial \mathscr{L}}{\partial q},
$$

where $\mathscr{L}=\mathscr{L}(q, Q)$ is the generating function $\mathscr{L}=M^{n} \times M^{n} \rightarrow \mathbb{R}$ (see [13]). To find $(P, Q)$ for given $(p, q)$ we need to solve the system $(2.1)$, which in general has no unique solution. Such mappings correspond to the Lagrangian systems with discrete time, where $\mathscr{L}$ plays the role of the Lagrangian (see e.g. [14]).

Let us give some natural definitions. General multiple-valued mapping or correspondence $\Phi: M \rightarrow M$ is determined by its graph $\Gamma_{\Phi} \subset M \times M$. The point $y \in M$ is called the image of $x \in M$ under the correspondence $\Phi$ if $(x, y) \in \Gamma_{\Phi}$. More generally, $y$ is the image of $x$ under the $k^{\text {th }}$ iteration of $\Phi$ if there exists a sequence $\left(x=x, x_{1}, \ldots, x_{k}=y\right)$, such that $\left(x_{i-1}, x_{i}\right) \in \Gamma_{\Phi}, i=1, \ldots, k$. If $M=M^{2 n}$ is some symplectic manifold with the structure $\omega$, then we will call the correspondence $\Phi$ as symplectic if its graph $\Gamma_{\Phi}$ is a Lagrangian submanifold of $M^{2 n} \times M^{2 n}$ with the induced structure

$$
\Omega=\pi_{1}^{*} \omega-\pi_{2}^{*} \omega
$$

$\pi_{i}: M^{2 n} \times M^{2 n} \rightarrow M^{2 n}$ are the projections on the first and second factors correspondingly. The function $F$ on the manifold $M^{2 n}$ is called the integral of $\Phi$ if the function $\pi_{i}^{*} F-\pi_{2}^{*} F$ after the restriction to $\Gamma_{\Phi}$ becomes zero.

We will say that the symplectic correspondence $\Phi: M^{2 n} \rightarrow M^{2 n}$ is integrable (in Liouville's sense) if there exist $n$ independent integrals $F_{1}, \ldots, F_{n}$ being in involution $\left\{F_{i}, F_{j}\right\}=0$. The common nondegenerate compact level of these integrals

$$
M_{c}=\left\{x \in M^{2 n}: F_{i}(x)=c_{i}\right\}
$$

must be the finite set of the tori $T_{1}^{n}, \ldots, T_{p}^{n}$ for the same reasons as in the usual Liouville theorem (see [13]).

1 The author is grateful to A. Eremenko, who called his attention to the paper [10] and explained how its results can be used to answer one of the questions posed in [4] 
Let us consider the numbers of different images of some point $x$ under the $k^{\text {th }}$ iteration of $\Phi$,

$$
N_{x}^{\Phi}(k)=\# \Phi^{(k)}(x) .
$$

If $N_{x}^{\Phi}(1)=m>1$ then it is quite natural to expect that these numbers will grow exponentially

$$
N_{x}^{\Phi}(k) \approx m^{k}
$$

It turns out that for the integrable correspondences they grow much slower.

Theorem 2. The numbers of the different images of the point under the $k^{\text {th }}$ iteration of the integrable symplectic correspondence are growing no more then some polynomial on $k$. More precisely, if $p$ is the number of the components of $M_{c}$, containing the point $x$, and $m$ is the maximal number of images of $\Phi$ on the level $M_{c}$, then there exists some constant $C$, independent of $k$, such that

$$
N_{x}^{\Phi}(k)<C k^{m p-1} .
$$

Proof is based on the corresponding discrete version of Liouville theorem (see [3]). The idea becomes clear already in the case $p=1$, i.e. when $M_{c} \approx T^{n}$. On the level $M_{c}$ we have the action of the abelian group $\mathbb{R}^{n}$, generating by the Hamiltonian flows with the Hamiltonians $F_{1}, \ldots, F_{n}$. Our mapping $\Phi$ commutes with this action and therefore should determine some set of shifts

$$
\Phi(x)=\{x+a\}, \quad a \in A \subset \mathbb{R}^{n} .
$$

Such mappings $\Phi: T^{n}=\mathbb{R}^{n} / L \rightarrow T^{n}$ we will call as multiple-valued shifts and denote as $t_{A}$. Now the numbers $N_{x}^{\Phi}(k)$ are easily calculated

$$
N_{k}^{\Phi}(k) \leq C_{m+k-1}^{k}=\frac{(k+1) \ldots(k+m-1)}{(m-1) !} \leq C(m) k^{m-1}
$$

where $m=|A|=N_{x}^{\Phi}(1)$.

The following picture demonstrates the difference between integrable and general situations
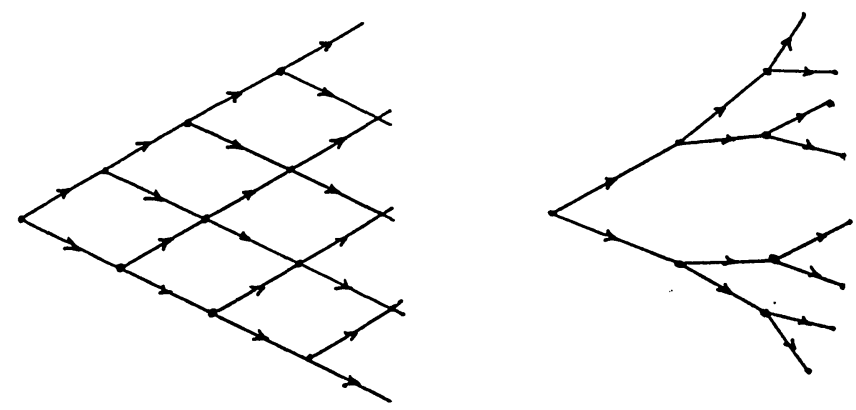

Fig. 1

For the several components of $M_{c} p>1$ let us consider two of them, say $T_{1}$ and $T_{2}$, connected by correspondence $\Phi$. They can be represented as quotients $T_{i}=\mathbb{R}^{n} / L_{i}$, where the lattices $L_{i}$ are the corresponding stationary subgroups for the action of $\mathbb{R}^{n}$. Let us define also the lattice $L_{12}$,

$$
L_{12}=\left\{b \in \mathbb{R}^{n}: \Phi(x+b) \equiv \Phi(x)\right\}
$$


or, equivalently,

$$
L_{12}=\left\{b \in \mathbb{R}^{n}: \Phi(x) \equiv \Phi(x)+b\right\}, \quad x \in T_{1}, \quad \Phi(x) \in T_{2} .
$$

It contains $L_{1}$ and $L_{2}$ as a sublattice. Considering corresponding torus $T_{12}=\mathbb{R}^{n} / L_{12}$ we have the following commutative diagram

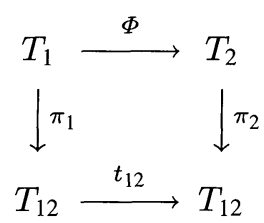

where $t_{12}$ is some multiple-valued shift, so our correspondence $\Phi$ has the form

$$
\Phi=\pi_{2}^{-1} \circ t_{A} \circ \pi_{1} \text {. }
$$

Now let us take all tori of $M_{c}$, which are connected with the given torus $T_{1}$, containing the point $x$, by the correspondence $\Phi$ and its iterations, let it be $T_{1}, \ldots, T_{q}, q \leq p$. There exists the lattice $L$, which contains the lattices $L_{i j}$ described above for all possible $1 \leq i, j \leq q$. On the corresponding torus $T=\mathbb{R}^{n} / L$ we have the multiplevalued shift, which is the projection of $\Phi$. It is easy to see that it consists of no more than $m q$ usual shifts. It means that the numbers of the images for the iterations after such a projection grow as

$$
N(k) \leq C k^{m q-1} \leq C k^{m p-1} .
$$

But these numbers differ from ones we need only by some bounded factor. The proof is finished.

In the algebraic situation when the Liouville tori are the real parts of some abelian varieties, the role of the number $p$ plays the number of irreducible components of $M_{c}$. In particular, for the irreducible $M_{c}$ we have the inequality

$$
N(2) \leq \frac{m(m+1)}{2}<m^{2}, \quad \text { if } \quad m>1
$$

It can be used as an effective test for such integrable systems. The examples of such systems can be found in [14].

\section{The Dynamics of the Algebraic Correspondences $\mathbb{C} P^{1} \rightarrow \mathbb{C} P^{1}$ and Yang-Baxter Equation}

Let us consider now the correspondences $\Phi: \mathbb{C} P^{1} \rightarrow \mathbb{C} P^{1}$, determined by the polynomial equation

$$
\Phi(x, y)=0 .
$$

We say that $\Phi$ is $m-n$ correspondence if the degrees of $\Phi(x, y)$ with respect to $x$ and $y$ are $n$ and $m$ correspondingly. It means that in the general case the point has $m$ images and $n$ preimages under the mapping $\Phi$. The composition of two correspondences $\Phi(x, y)=0$ and $\Psi(x, y)=0$ can be found with the help of resultant.

Let $N^{\Phi}(k)$ be the number of the different images of $k^{\text {th }}$ iteration of $\Phi$ for the general point $x$

$$
N^{\Phi}(k)=\# \Phi^{(k)}(x) .
$$


The problem we are interested in is how these numbers are growing and when this growth is polynomial on $k$.

This problem seems to be very difficult but very interesting for many reasons. One follows from the previous discussion of the symplectic case and concerns the general problem of integrability for the multiple-valued mappings. The second motivation goes from the theory of the Yang-Baxter equation and quantum integrable systems (see below).

We begin with the simplest case of 2-2 correspondences, given by the biquadratic equation

$$
\Phi(x, y)=\sum_{0 \leq i, j \leq 2} a_{i j} x^{i} y^{j}=0 .
$$

Some results about the dynamics of these mapping can be found in [15]. The numbers $N^{\Phi}(k)$ for the general coefficients $a_{i j}$ grow exponentially

$$
N^{\Phi}(k)=2^{k} \text {. }
$$

The reason for the slow growth is the glueing of the images for some iterations of $\Phi$. Let us consider the case when it happens already for the second iteration of $\Phi: N^{\Phi}(2)<2^{2}=4$. Two possible situations are pictured in Fig. 2 .
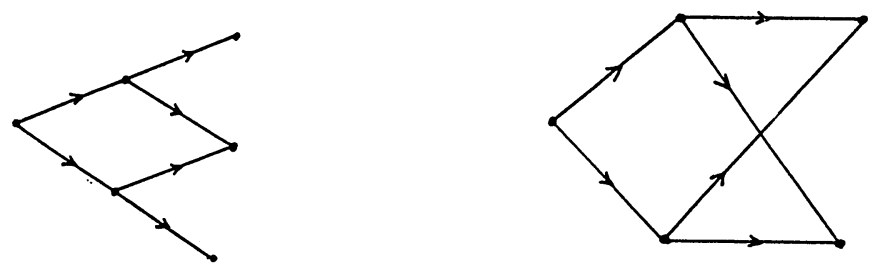

Fig. 2

Theorem 3. The glueing of the images for the second iteration has a place only for the following 2-2 correspondences:

1) symmetric correspondence $\Phi(x, y)=\Phi(y, x)$,

$$
\begin{aligned}
\Phi(x, y)= & a_{22} x^{2} y^{2}+a_{12} x y(x+y)+a_{20}\left(x^{2}+y^{2}\right) \\
& +a_{11} x y+a_{01}(x+y)+a_{00}=0 ;
\end{aligned}
$$

2) reducible correspondence

$$
\Phi(x, y)=\left(\alpha_{1} x y+\beta_{1} x+\gamma_{1} y+\delta_{1}\right)\left(\alpha_{2} x y+\beta_{2} x+\gamma_{2} y+\delta_{2}\right)=0,
$$

corresponding to the commuting transformations $x \rightarrow y=-\frac{\beta_{i} x+\delta_{i}}{\alpha_{i} x+\gamma_{i}}(i=1,2)$;

3) the correspondence

$$
\Phi(x, y)=x y(x+\varepsilon y)+b=0, \quad \varepsilon^{3}=1, \quad \varepsilon \neq 1 ;
$$

4) the correspondence $\Phi$ of the form

$$
\Phi(x, y)=a x^{2} y^{2}+b x^{2}+c y^{2}+d=0 ;
$$

and for the correspondences, which are equivalent to these ones up to the projective transformations of the independent variable. 
Before the proof let us make some comments.

A symmetric biquadratic relation of the form (3.1) plays an important role in Euler's proof of the addition law for the elliptic integrals. Chasles was the first who realized it as the multiple-valued mapping in the geometrical situation pictured in Fig. 3 and related it to the "great" Poncelet theorem (see [16])

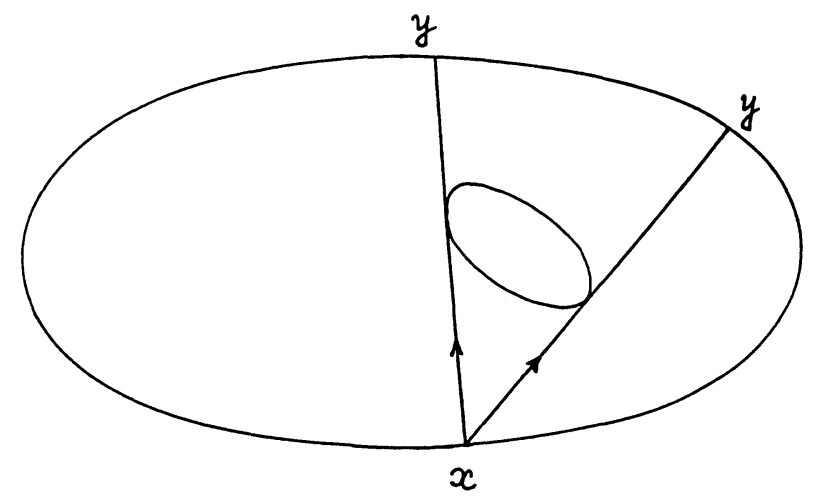

Fig. 3

The curves pictures here are conics, $x$ is the rational parameter on one of them. We will call the correspondence (3.1) a Euler-Chasles correspondence. To describe its dynamics notice that Eq. (3.1) determines in general a position on the elliptic curve $\Gamma$, which has the symmetry $\sigma:(x, y) \rightarrow(y, x)$. There exists also the second involution $\tau$, changing the lists of $y$, which is a 2-valued function of $x$. The composition of these involutions $\sigma \circ \tau$ corresponds to the shift of $\Gamma: z \rightarrow z+a$ for some $a$. So we have the commutative diagram

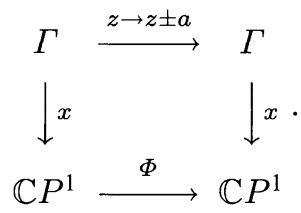

In other words there exists some elliptic function $\varphi(z)$ (the function $x$ on $\Gamma$ ) of the second order, such that if $x=\varphi(z)$, then $y=\varphi(z \pm a)$. This consideration is the slight variation of one from the paper by Griffiths [17], devoted to Poncelet theorem. The decomposition of the mapping into the product of two involutions often happens in the discrete dynamics (see e.g. $[12,14]$ ).

The reducible correspondence needs not any comments.

The mapping (3.3) corresponds to the transformations $z \rightarrow \varepsilon z \pm a$ for the elliptic curves given by (3-3) and having the parameter $\tau=\frac{1+\sqrt{3} i}{2}, a=\frac{2+\varepsilon}{3}$. Indeed it follows from the addition theorem for the Weierstrass $\mathscr{P}$-function that the pairs $(\mathscr{P}(z), \mathscr{P}(\varepsilon z \pm a))$ satisfy the equation

$$
x^{2} y^{2}+g_{3}(x+\varepsilon y)=0,
$$

which after the change $x \rightarrow \frac{1}{x}, y \rightarrow \frac{1}{y}$ goes to the (3.3). 
The correspondence (3.4) is semiconjugate to the usual projective mapping: after the noninvertible change $u=x^{2}$ the relation (3-4) has the form

$$
a u v+b u+c v+d=0 \text {, }
$$

which describes the mapping $u \rightarrow-\frac{b u+d}{a u+c}$.

Proof of Theorem 3. Let us begin with the case pictured on the left scheme of Fig. 2: $N(2)=3$. In this case we have the well-defined mapping $x \rightarrow z$ (see Fig. 4)

Fig. 4

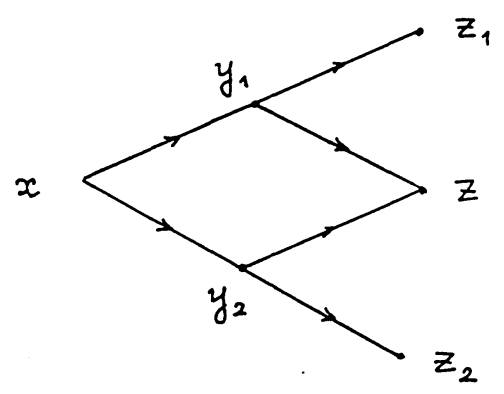

After appropriate projective transformations we have two possibilities

1) $z=x+a$; 2) $z=\lambda x$.

In the first case the curve $\Gamma_{\Phi}$ must be invariant under the transformation $\sigma:(x, y) \rightarrow$ $(y, x+a)$, and therefore under $\sigma^{2}:(x, y) \rightarrow(x+a, y+a)$. If $a \neq 0$ such a curve has to be a pair of the straight lines of the form $y-x=$ const. So $\Phi$ determines two shifts and belongs to the family (2). If $a=0$ then $\Gamma_{\Phi}$ has a symmetry $\sigma:(x, y) \rightarrow(y, x)$. It means that $\Phi(x, y)= \pm \Phi(y, x)$. If $\Phi(x, y)=\Phi(y, x)$ we have Euler-Chasles correspondence. Otherwise $\Phi$ has the form

$$
x y(x-y)+b(x-y)=0
$$

and belongs to the type (3.3).

In the case $z=\lambda x, \lambda \neq 1$ the curve $\Gamma_{\Phi}$ is invariant under the transformation $\sigma(x, y)=(y, \lambda x)$ and its iterations. In particular, for every point $(x, y) \in \Gamma_{\Phi}$ the points $\sigma^{2 k}(x, y)=\left(\lambda^{k} x, \lambda^{k} y\right)$ also belong to $\Gamma_{\Phi}$ for all $k \in \mathbb{N}$. If $\lambda^{k} \neq 1$ for $k=2,3,4$ then we have more than four common points of $\Gamma$ with the corresponding straight line $\alpha x+\beta y=0$, and therefore $\Gamma$ must be the union of two such lines

$$
\left(\alpha_{1} x+\beta_{1} y\right)\left(\alpha_{2} x+\beta_{2} y\right)=0 .
$$

The investigation of the cases $\lambda^{k}=1, k=2,3,4$ adds the only new correspondence (3.3) (for $k=3$ ).

When $N(2)=2$ (left diagram of Fig. 2) we have well-defined involution $\sigma: \mathbb{C} P^{\prime} \rightarrow$ $\mathbb{C} P^{1}$ (see Fig. 5)

In the appropriate coordinate it has the form $\sigma(x)=-x$ and the correspondence $\Phi$ must be the mapping of pairs $\Phi:( \pm x) \rightarrow( \pm y)$ (compare with [15]). All such correspondences have the form (3.4). The proof is finished.

Corollary. The glueing of the images of the second iteration implies for 2-2 correspondences the explicit description of the dynamics and no more than linear growth of $N^{\Phi}(k)$. 
Fig. 5

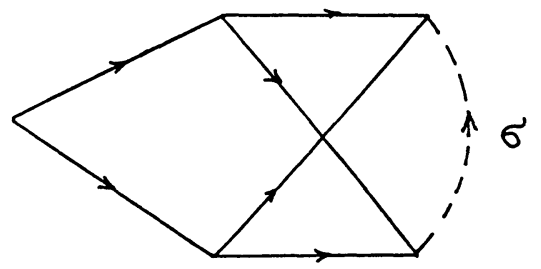

In the paper [3] the correspondences whose dynamics for the first two iterations has the same scheme as for a multiple-valued shift on some torus, are called abelian. If it is true for all iterations we will call such correspondences abelian in the strong sense. So among all 2-2 correspondences only those pointed out in the first three cases of Theorem 3 are abelian.

To describe all abelian mapping is an interesting open problem. It is connected with the following problem, investigated by Julia, Faton, and Ritt [18-20]: to describe all commuting rational (polynomial) mappings of $\mathbb{C} P^{1}$. Under some assumptions all such mapping turn out to be connected with the transformation law for the elliptic functions and its elementary degenerations (see [18-21, 1]). It is easy to see that the reducible correspondence

$$
(y-f(x))(y-g(x))=0
$$

is abelian if $f \circ g=g \circ f$. One more demonstration of the connection of the problem about commuting mappings and the problem of growth of $N(k)$ is given by the following simple proposition.

Let $\Phi^{-1}$ denote the correspondence whose graph is symmetric to $\Gamma_{\Phi}$ according to the diagonal $x=y$,

$$
\Phi^{-1}(x, y)=\Phi(y, x)=0 .
$$

Notice that $\left(\Phi^{-1}\right)^{-1}$ and the composition $\Phi^{-1} \circ \Phi$ as well as $\Phi \circ \Phi^{-1}$ in general is some multiple-valued, but not identical mapping.

Proposition. The condition

$$
\Phi^{-1} \circ \Phi=\Phi \circ \Phi^{-1}
$$

for 2-2 correspondence $\Phi$ implies the glueing of the images of the second iteration of $\Phi$ and therefore leads to the list of Theorem 3.

The proof follows from the simple analysis of the possible schemes of the dynamics for such mappings.

The general problem about the commuting correspondences is far from the complete investigation in contrast with the case of rational mappings. The following consideration shows that here we have some principally new examples.

Let $\Phi_{n}^{J}(x, y)=0$ be the modular equation, which is satisfied by the pairs $(x, y)$, $x=J(z), y=J(n z), J(z)$ is modular function. This equation plays a very important role in the theory of elliptic curves (see e.g. [21]). In the simplest nontrivial case $n=2$ it has the following form:

$$
\begin{aligned}
x^{3} & +y^{3}-x^{2} y^{2}+2^{4} \cdot 3 \cdot 31 x y(x+y)-2^{4} \cdot 3^{4} \cdot 5^{3}\left(x^{2}+y^{2}\right) \\
& +3^{4} \cdot 5^{3} \cdot 4027 x y+2^{8} \cdot 3^{7} \cdot 5^{6}(x+y)-2^{12} \cdot 3^{9} \cdot 5^{9}=0 .
\end{aligned}
$$


Using the properties of these equations, it is easy to prove that the correspondences determined by them for the mutually prime $m$ and $n$ commute

$$
\Phi_{m}^{J} \circ \Phi_{n}^{J}=\Phi_{n}^{J} \circ \Phi_{m}^{J}=\Phi_{m n}^{j}
$$

The problem about commuting correspondences appeared in the paper by Krichever [22], devoted to the solution of the quantum Yang-Baxter (YB) equation. One of the crucial steps in this paper consists of the construction of some correspondence for the certain solutions of the YB equation. The further investigation shows that only correspondences (3.1) and (3.4) are possible, which leads to the final classification of the considered solutions of the YB equation. Euler-Chasles mapping corresponds to the well-known Baxter solution [23], the correspondence (4) - to the solution found by Felderhof.

It is interesting that the important role of the YB equation in the problem of growth for the related algebraic objects, was underlined by Faddeev and his collaborators from the very beginning of the theory of quantum integrable systems and quantum groups. For example, Sklyanin algebra [24] is the algebra with the quadratic relations, which grows precisely like the commutative algebra of the polynomials. Notice that this growth coincides with the growth of the numbers $N_{x}(k)$ for the corresponding multiple-valued shift. The analogy with the notion of the abelian correspondence seems to be clear.

From this point of view the following generalizations of Euler-Chasles correspondence seems to be important (see [1]).

Let $G$ be the complex simple Lie algebra of rank $n, H$ is its Cartan subalgebra, $L$ is the lattice in $H$, dual to the lattice, generated by the fundamental weights $\omega_{1}, \ldots, \omega_{n}$ (see [25]). Determine the abelian variety $M_{G}$ as the quotient

$$
M_{G}=H / L+\tau L
$$

where $\tau \in \mathbb{C}, \operatorname{Im} \tau>0$. It is the product of the elliptic curves with the parameter $\tau$, on which the natural action of Weyl group $W$ is defined. As it was proven by Looijenga $[26,28]$. Bernstein and Schwarzman [27] the quotient $M_{G} / W$ is isomorphic to the "weighted"projective space $\mathbb{C} P^{n}$. So for any $W$-invariant finite set $A$ we can determine the correspondences $\Phi_{G, A}$ from the following commutative diagram

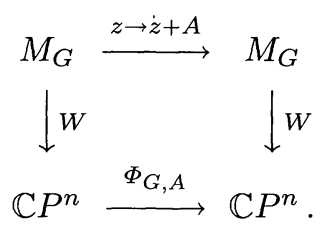

For the case $G=A_{1}, A=\{ \pm a\}, W=\mathbb{Z}_{2}$ we have a Euler-Chasles correspondence of the form (3.1), so $\Phi_{G, A}$ can be considered as its multidimensional generalizations. Notice that $\Phi_{G, A}$ and $\Phi_{G, B}$ commute

$$
\Phi_{G, A} \circ \Phi_{G, B}=\Phi_{G, B} \circ \Phi_{G, A}
$$

and the numbers of the images for its iterations $N(k)$ grow polynomially. Their dynamics is clear from the definition. To understand what the quantum analogues of these correspondences are is the problem under investigation. 


\section{References}

1. Veselov, A.P.: What is an integrable mapping. In the book "What is integrability". Zakharov, V.E. (ed.). Berlin, Heidelberg, New York: Springer 1991

2. Arnold, V.I.: Dynamics of the complexity of intersections. Bol. Mat. Soc. Bras., 1991. Dynamics of intersections. In the book "Analysis et cetera." Research papers published in honour of J. Moser's $60^{\text {th }}$ birthday. Rabinowitz, P., Zehnder, E. (ed.). New York: Academic Press 1990

3. Veselov, A.P.: On the growth of the numbers of images of the point under the iterations of multiple-valued mapping. Mat. Zametki, 49, (2) (1991) (Russian)

4. Veselov, A.P.: Cremona group and dynamical systems. Mat. Zametki, 45, (3), 118-120 (1989) (Russian)

5. Mikhailov, A.V., Shabat, A.B., Yamilov, R.I.: The symmetry approach to the classification of nonlinear equations. The complete list of the integrable systems. Russ. Math. Surv. 42, (4), 3-53 (1987)

6. Jung, H.: Über ganze birationale Transformationen der Ebene. J. Reine Angew. Math., 184, 161-172 (1942)

7. Wright, D.: Abelian subgroups of $\operatorname{Aut}_{k}(k[x, y])$ and applications to actions on the affine plane. Ill. J. Math., 23, (4), 579-633 (1979)

8. Moser, J.: On the integrability of area preserving Cremona mappings near an elliptic fixed point. Bol. Soc. Mat. Mexicana, 176-180 (1960)

9. Hénon, M.: A two dimensional mapping with a strange attractor. Commun. Math. Phys., 50, 69-77 (1967)

10. Friedland, S., Milnor, J.: Dynamical properties of plane polynomial automorphisms. Ergod. Theory Dyn. Systems, 9, 67-99 (1989)

11. Veselov, A.: Integrable mappings. Russ. Math. Surv. 46(5), 3-45 (1991) (Russian)

12. Moser, J.: On quadratic symplectic mappings. Preprint of FIM (ETH, Zürich), June 1991

13. Arnold, V.I.: Mathematical methods of classical mechanics. Berlin, Heidelberg, New York: Springer 1978

14. Moser, J., Veselov, A.P.: Discrete versions of some classical integrable systems and factorization of matrix polynomials. Commun. Math. Phys. 139, 217-243 (1991)

15. Bullett, S.: Dynamics of quadratic correspondences. Nonlinearity 1, 27-50 (1988)

16. Berger, M.: Geometry I, II. Berlin, Heidelberg, New York: Springer 1987

17. Griffiths, P.: Variations on a theorem of Abel. Invent. Math. 35, 321-390 (1976)

18. Julia, G.: Mémoire sur le permutabilité des fractions rationelles. Ann. Sci. Ecole Norm. Super. 39, 131-215 (1922)

19. Fatou, P.: Sur le fonctions qui admettent plusieurs théorèmes de multiplication. C.R. Acad. Sci. Paris, 173, 571-573 (1921)

20. Ritt, J.: Permutable rational functions. trans. Am. Math. Soc. 25, 399-448 (1923)

21. Lang, S.: Elliptic functions. Berlin, Heidelberg, New York: Springer 1987

22. Krichever, I.M.: Baxter equation and algebraic geometry. Funct. Anal. Appl. 15, (3) (1981)

23. Baxter, R.: Exactly solvable models in statistical mechanics. New York: Academic Press 1982

24. Sklyanin, E.: On the certain algebraic structures related to the Yang-Baxter equation. Funct. Anal. Appl., 16, (4) (1982)

25. Bourbaki, N.: Groupes et Algèbres de Lie. Chap. 6. Paris: Hermann 1969

26. Looijenga, E.: Root systems and elliptic curves. Invent. Math. 38, 17-33 (1976)

27. Bernstein, I.N., Schwarzman, O.V.: Chevalley's theorem for complex cristallographic Coxeter groups. Funct. Anal. Appl. 12, 79-80 (1978)

28. Looijenga, E.: Invariant theory for generalized root systems. Invent. Math. 61, 1-32 (1980) 
